\title{
The Hypercomplex Solution of the Dirac Equation
}

\author{
Konstantin Karplyuk ${ }^{1}$, Oleksandr Zhmudskyy ${ }^{2, *}$ \\ ${ }^{1}$ Department of Radiophysics, Taras Shevchenko University, Academic Glushkov prospect 2, building 5, Kyiv 03122, Ukraine \\ ${ }^{2}$ Department of Physics, University of Central Florida, 4000 Central Florida Blvd. Orlando, FL, 32816 \\ *Corresponding Author: Oleksandr.Zhmudskyy@ucf.edu,ozhmudsky@physics.ucf.edu
}

Copyright (C)2013 Horizon Research Publishing All rights reserved.

\begin{abstract}
It is shown that the hypercomplex Dirac equation describes the system of connected fields: 4-scalar, 4-pseudoscalar, 4-vector, 4-pseudovector and antisymmetric 4-tensor second rank field. If mass is assumed to be zero this system splits into two subsystems. Equations containing tensor, scalar and pseudoscalar fields coincide with Maxwell equations complemented by scalar and pseudoscalar fields. This system describes the electrodynamics of non-conserved charges. The scalar and pseudoscalar fields are generated only by the non-conserved charges - electric and hypothetical magnetic. The influence of these fields on the charged particles is very unusual - it causes a change of their rest mass. This allows us to give a new look at the Wigner paradox and mechanism of mass renormalization.
\end{abstract}

Keywords Dirac Equation, Hypercomplex numbers

\section{Introduction}

It is known that Maxwell equations can be written down as the massless Dirac equation if the elements of dirac column are represented as $\psi_{i}=a_{j} E_{j}+b_{k} B_{k}$. Here $a_{j}$ and $b_{k}$ are some coefficients and $E_{j}$ and $B_{k}$ are components of the electric and magnetic fields. Such representation can be done in several ways so different authors have different combination for $a_{j} E_{j}+b_{k} B_{k}$. The first versions of such a notation were proposed in $[1,2,3]$ and later this subject was developed in several hundreds of works.

There is another method to use the Dirac equation for the derivation of new equations. According to this method the $\psi$-function is considered as a hypercomplex number built on 16 independent dirac matrices $\Gamma^{i}$. We will study this approach below and we will show that presentation of the $\psi$-function as $\psi=a_{i} \Gamma^{i}$ results in the system of equations which describes the set of mutually connected fields of different tensor dimensional representation.

The obtained system of equations contains some known equations. Among them, the Maxwell equa- tions complemented by the scalar field $\epsilon$. Such modified Maxwell equations are used for electromagnetic field quantization [4]. The introduction of a scalar field however always was considered as a purely formal technique necessary for implementation of the canonical quantization procedure. The physical nature of the scalar field has not been studied. It turns out however that extended in such a way, the Maxwell equations describe the electrodynamics of the non-conserved charges. A scalar $\epsilon$-field is generated only by the non-conserved charges. It can spread out forming a wave together with the longitudinal electric field. Its influence on the charged particles is very unusual - it changes the rest mass of these particles. In particular, self-action of this field on the non-conserved charges, generating them, results in transformation of their rest energy (i.e. rest mass) into the energy of their electric field and vice versa. It becomes possible to observe in what way part of the mass of an initially uncharged particle transforms into electromagnetic mass while a charge is generated for this particle. Thus we have an opportunity to compare the physical mechanism of renormalization with the idea of such mass renormalization. It is possible as well to avoid contradictions during consideration of processes with the non-conserved charges mentioned by Wigner. Below we will consider these and other questions in more detail.

\section{The hypercomplex solutions of the Dirac Equation}

It is known that the system of hypercomplex numbers with 16 units can be built on dirac matrices as follows:

$$
\gamma^{\mu} \gamma^{\nu}+\gamma^{\nu} \gamma^{\mu}=2 \eta^{\mu \nu}
$$

Here, $\eta^{\mu \nu}$ is the matrix of Minkowski metrical tensor, $\eta^{\mu \nu}=\operatorname{diag}(1,-1,-1,-1)$. The formal solution of the Dirac equation

$$
\left(i \gamma^{\nu} \partial_{\nu}-\frac{m c}{\hbar}\right) \psi=0
$$

may be treated as a hypercomplex number of this system. From a group point of view such a solution will belong to the reduced presentation of the Lorentz group. 
The importance of such solutions was pointed out by Lev [22]. Let us discuss this possibility.

We will look for a solution of (1) of the form

$$
\begin{array}{r}
\psi=\epsilon I+i V_{\nu} \gamma^{\nu}+F_{0 k} \gamma^{0} \gamma^{k}+F_{23} \gamma^{2} \gamma^{3}+F_{31} \gamma^{3} \gamma^{1} \\
+F_{12} \gamma^{1} \gamma^{2}+i U_{0} \gamma^{1} \gamma^{2} \gamma^{3}+i U_{1} \gamma^{0} \gamma^{2} \gamma^{3} \\
+i U_{2} \gamma^{0} \gamma^{3} \gamma^{1}+i U_{3} \gamma^{0} \gamma^{1} \gamma^{2}+\beta \gamma^{0} \gamma^{1} \gamma^{2} \gamma^{3}
\end{array}
$$

Here, $I$ is the unity matrix, the Latin indices take on values 1,2,3, Greek indices - 0,1,2,3. Repeated Latin indices are summed from 0 to 3 . Repeated Greek indices are summed from 0 to 4 . Substituting $\psi$ into (1) and equating the coefficients of each of the 16 independent matrices to zero we get 16 equations:

$$
\begin{aligned}
\frac{\partial \epsilon}{\partial x^{\nu}}-\frac{\partial F_{\nu}^{\mu}}{\partial x^{\mu}}-\varkappa V_{\nu} & =0 \\
\frac{\partial \beta}{\partial x^{\nu}}+\frac{\eta_{\nu \alpha}}{2} \varepsilon^{\alpha \beta \gamma \delta} \frac{\partial F_{\gamma \delta}}{\partial x^{\beta}}-\varkappa U_{\nu} & =0 \\
\frac{\partial V_{\alpha}}{\partial x^{\beta}}-\frac{\partial V_{\beta}}{\partial x^{\alpha}}+\eta_{\alpha \nu} \eta_{\beta \mu} \varepsilon^{\nu \mu \gamma \delta} \frac{\partial U_{\gamma}}{\partial x^{\delta}}-\varkappa F_{\alpha \beta} & =0 \\
\frac{\partial V^{\nu}}{\partial x^{\nu}}+\varkappa \epsilon & =0 \\
\frac{\partial U^{\nu}}{\partial x^{\nu}}+\varkappa \beta & =0
\end{aligned}
$$

Here, $\varkappa$ is the Compton wave number, $\varkappa=2 \pi / \lambda_{k}=$ $m c / \hbar$.

Equations (3)-(7) describe the system of connected fields: 4-scalar $\epsilon$, 4-pseudoscalar $\beta$, 4-vector $V_{\nu}, 4$ pseudovector $U_{\nu}$ and an antisymmetric 4-tensor second rank field $F_{\mu \nu}$. In the case of zero-point mass $(\varkappa=0)$ this system splits into two independent subsystems. Equations $(3),(4)$ in this case coincide with homogeneous Maxwell equations complemented by scalar filed $\epsilon$ and pseudoscalar field $\beta$. Equations (5)-(7) describe the system of connected 4 -vector and 4-pseudovector fields.

Equations (3)-(7) are homogeneous. We put the sources of the fields into right sides of the (3)-(7) in order to study connections between the fields and their sources as well as the influence of the fields on corresponding charges and currents. In three-dimensional notation a suitable system looks like

$$
\begin{aligned}
\frac{1}{c} \frac{\partial \epsilon}{\partial t}+\nabla \cdot \boldsymbol{E}-\varkappa V^{0} & =\zeta c \rho_{e}, \\
\frac{1}{c} \frac{\partial \boldsymbol{E}}{\partial t}-\nabla \times c \boldsymbol{B}+\nabla \epsilon+\varkappa \boldsymbol{V} & =-\zeta \boldsymbol{j}_{e}, \\
-\frac{1}{c} \frac{\partial \beta}{\partial t}+\nabla \cdot c \boldsymbol{B}+\varkappa U^{0} & =-\zeta c \rho_{m}, \\
\frac{1}{c} \frac{\partial c \boldsymbol{B}}{\partial t}+\nabla \times \boldsymbol{E}-\nabla \beta-\varkappa \boldsymbol{U} & =\zeta \boldsymbol{j}_{m}, \\
\frac{1}{c} \frac{\partial V^{0}}{\partial t}+\nabla \cdot \boldsymbol{V}+\varkappa \epsilon & =\zeta s, \\
\frac{1}{c} \frac{\partial \boldsymbol{V}}{\partial t}-\nabla \times \boldsymbol{U}+\nabla V^{0}-\varkappa \boldsymbol{E} & =-\zeta \boldsymbol{k}, \\
\frac{1}{c} \frac{\partial \boldsymbol{U}}{\partial t}+\nabla \times \boldsymbol{V}+\nabla U^{0}+\varkappa c \boldsymbol{B} & =\zeta \boldsymbol{l}, \\
\frac{1}{c} \frac{\partial U^{0}}{\partial t}+\nabla \cdot \boldsymbol{U}+\varkappa \beta & =-\zeta p .
\end{aligned}
$$

Identification of three-dimensional and four-dimensional coordinates of vectors and tensors are as usuall: $\boldsymbol{E}=$ $\left(F_{01}, F_{02}, F_{03}\right), \quad c \boldsymbol{B}=\left(-F_{23},-F_{31},-F_{12}\right), \quad \boldsymbol{V}=$ $\left(V^{1}, V^{2}, V^{3}\right), \boldsymbol{U}=\left(U^{1}, U^{2}, U^{3}\right)$. Equations (8)-(15) are written down in SI system but instead of constants $\varepsilon_{0}$ and $\mu_{0}$ we used velocity of light $c=1 / \sqrt{\varepsilon_{0} \mu_{0}}$ and resistance of the vacuum $\zeta=\sqrt{\mu_{0} / \varepsilon_{0}}$. This form of notation as applied to Maxwell equations allows us to use all the advantages of the Gaussian unit system, remaining at the same time in SI unit system.

In the system (8)-(15): $\rho_{e}$ and $\boldsymbol{j}_{e}$ are the densities of electric charges and currents, $\rho_{m}$ and $\boldsymbol{j}_{m}$ are the densities of hypothetical magnetic charges and currents. The nature of the other sources is to be determined. By analogy with an electric current $j_{e}^{\mu}=e \bar{\psi} \gamma^{\mu} \psi$ we can introduce bilinear combinations for the other sources, $s=e \bar{\psi} \psi, k^{i}=e \bar{\psi} \gamma^{0} \gamma^{i} \psi, l^{i}=e \bar{\psi} \gamma^{j} \gamma^{k} \psi, p=e \bar{\psi} \gamma^{5} \psi$, $j_{m}^{\mu}=e \bar{\psi} \gamma^{\mu} \gamma^{5} \psi$. The investigation of such a supposition helps us to find out why these bilinear combinations are not the sources of the fields and do not generate interactions like the $j^{\mu}=e \bar{\psi} \gamma^{\mu} \psi$.

The fields can be expressed using potentials:

$$
\begin{aligned}
\epsilon & =\frac{1}{c} \frac{\partial \varphi}{\partial t}+\nabla \cdot c \boldsymbol{A}+\varkappa S, \\
\boldsymbol{E} & =-\frac{1}{c} \frac{\partial c \boldsymbol{A}}{\partial t}-\nabla \varphi+\nabla \times \boldsymbol{\Pi}+\varkappa \boldsymbol{\theta}, \\
c \boldsymbol{B} & =\frac{1}{c} \frac{\partial \boldsymbol{\Pi}}{\partial t}+\nabla \Pi^{0}+\nabla \times c \boldsymbol{A}+\varkappa \boldsymbol{\vartheta}, \\
\beta & =\frac{1}{c} \frac{\partial \Pi^{0}}{\partial t}+\nabla \cdot \boldsymbol{\Pi}-\varkappa \Psi, \\
V^{0} & =\frac{1}{c} \frac{\partial S}{\partial t}+\nabla \cdot \boldsymbol{\theta}-\varkappa \varphi, \\
\boldsymbol{V} & =-\frac{1}{c} \frac{\partial \boldsymbol{\theta}}{\partial t}+\nabla \times \boldsymbol{\vartheta}-\nabla S-\varkappa c \boldsymbol{A}, \\
U^{0} & =-\frac{1}{c} \frac{\partial \Psi}{\partial t}-\nabla \cdot \boldsymbol{\vartheta}-\varkappa \Pi^{0}, \\
\boldsymbol{U} & =\frac{1}{c} \frac{\partial \boldsymbol{\vartheta}}{\partial t}+\nabla \times \boldsymbol{\theta}+\nabla \Psi-\varkappa \boldsymbol{\Pi} .
\end{aligned}
$$

Substitution of these expressions into (8)-(15), yields to the following equations for the potentials:

$$
\begin{aligned}
\square \varphi+\varkappa^{2} \varphi & =\zeta c \rho_{e}, & \square c \boldsymbol{A}+\varkappa^{2} c \boldsymbol{A}=\zeta \boldsymbol{j}_{e}, \\
\square \Pi^{0}+\varkappa^{2} \Pi^{0} & =\zeta c \rho_{m}, & \square \boldsymbol{\Pi}+\varkappa^{2} \boldsymbol{\Pi}=\zeta \boldsymbol{j}_{m} \\
\square \boldsymbol{\theta}+\varkappa^{2} \boldsymbol{\theta} & =\zeta \boldsymbol{k}, & \square \boldsymbol{\vartheta}+\varkappa^{2} \boldsymbol{\vartheta}=\zeta \boldsymbol{l}, \\
\square S+\varkappa^{2} S & =\zeta s, & \square \Psi+\varkappa^{2} \Psi=\zeta p .
\end{aligned}
$$

Here, $\square$ is the d'Alambertian, $\square=c^{-2} \partial^{2} / \partial t^{2}-\triangle$.

As for the Maxwell electrodynamics it is possible to get conservation laws of energy and momentum from (8)-(15). The derivation of these conservation laws is presented in the appendix A.

Equations (8)-(15) include some well known systems of equations as particular cases. We get the Proka equations for the homogeneous system (24)-(27) if we also set up $\epsilon=\beta=\boldsymbol{U}=U^{0}=0$. In the case $\varkappa=0$, system (8)-(15) splits into two independent systems. One of these subsystems coincides with the Maxwell equations complemented by fields $\epsilon$ and $\beta$.

The main question to be answered about (8)-(15) is whether these equations have any reference to physical 
reality? In order to answer this we have to investigate the properties of these equations.

\section{Electrodynamics of the non- conserved charges}

It is known that the Maxwell equations describe only such electromagnetic processes in which electric charge is conserved. On this subject Feynman [5] wrote : "The laws of physics have no answer to the question: "What happens if a charge is suddenly created at this point what electromagnetic effects are produced"? No answer can be given because our equations say it doesn't happen. If it were to happen, we would need new laws, but we cannot say what they would be."

It turns out that (8)-(15) also describe, among others, processes with non-conserved electric charges. If $\varkappa=0$ these equations split into two independent systems. The first one ((8)-(11)) are the Maxwell equations complemented by the fields $\epsilon$ and $\beta$.

Let us eliminate for a while hypothetical magnetic charges. In order to do this we must set $\rho_{m}=0, \boldsymbol{j}_{m}=0$, $\beta=0$. In this case we have:

$$
\begin{aligned}
\frac{1}{c} \frac{\partial \epsilon}{\partial t}+\nabla \cdot \boldsymbol{E} & =\zeta \rho c, \\
-\frac{1}{c} \frac{\partial \boldsymbol{E}}{\partial t}+\nabla \times c \boldsymbol{B}-\nabla \epsilon & =\zeta \boldsymbol{j}, \\
\nabla \cdot c \boldsymbol{B} & =0, \\
\frac{1}{c} \frac{\partial c \boldsymbol{B}}{\partial t}+\nabla \times \boldsymbol{E} & =0 .
\end{aligned}
$$

As was noted by Heisenberg and Pauli [4], in order to quantize the electromagnetic field we can introduce the $\epsilon$-field into Maxwell equations. It is known that the application of canonical quantization procedure to the Maxwell equations has not succeeded, because a zero canonical momentum corresponds to the canonical coordinate $\varphi$. Introduction of the $\epsilon$-field which is just a canonical momentum for a coordinate $\varphi$ solves this problem. After implementation of quantization to (28)-(31) the mean value of the $\epsilon$-field is set to zero, $\langle\psi|\epsilon| \psi\rangle=0$. Thus the $\epsilon$-field is removed from consideration and we go back to the Maxwell equations. As the $\epsilon$-field plays only a formal role in this procedure its physical nature was not studied.

Some properties of the equations (28)-(31) discussed in $[6]$.

\subsection{Sources of $\epsilon$-field}

First of all, we need to find the source of the $\epsilon$-field. Let us take the derivative $c^{-1} \partial / \partial t$ of (28) and the divergence of (29), and add them:

$$
\square \epsilon=\zeta\left(\frac{\partial \rho}{\partial t}+\nabla \cdot j\right) .
$$

The right hand side of the (32) is a source of the $\epsilon$-field. It differs from zero only if the conservation law of electric charge is not valid,

$$
\frac{\partial \rho}{\partial t}+\nabla \cdot \boldsymbol{j} \neq 0
$$

Thus, the $\epsilon$-field is generated only by the non-conserved electric charges. Connection between non-conserving of the charge and massless of the photon discussed in ([7] - [21]).

The $\epsilon$-field can form a wave propagating jointly with the longitudinal electric field. The plane $\epsilon-\boldsymbol{E}$ wave has the form

$$
\begin{array}{r}
\epsilon=\epsilon_{0} \cos (\omega t-\boldsymbol{k} \cdot \boldsymbol{r}), \quad \boldsymbol{E}=\boldsymbol{k} \frac{c}{\omega} \epsilon_{0} \cos (\omega t-\boldsymbol{k} \cdot \boldsymbol{r}), \\
\boldsymbol{B}=0, \quad k^{2}=\frac{\omega^{2}}{c^{2}} .
\end{array}
$$

Unlike a transversal wave this wave does not contain the magnetic field. The electric field of such a wave is directed along the phase velocity, $\boldsymbol{E} \| \boldsymbol{k}$. We will illustrate the process of $\epsilon$-field birth by an example. Let us find the $\epsilon$-field generated by a spherical shell of radius $r_{0}$. Let us also assume that charge is distributed uniformly over the sphere and its time dependence $q(t)$ obeys an exponential law. We chose as an example a sphere with a finite radius, instead of point charge, to avoid infinity at $r=0$. The potential $\varphi$ of such a shell in the external region $r \geq r_{0}$ is determined by the retarded solution of (24):

$$
\varphi(r, t)=\frac{\zeta c}{4 \pi} \frac{1}{2 r r_{0}} \int_{r-r_{0}}^{r+r_{0}} q\left(t-\frac{x}{c}\right) d x .
$$

When the charge increases from 0 to $q_{0}$ according to:

$$
q(t)=\left\{\begin{array}{cc}
0 & t \leq 0 \\
q_{0}\left(1-e^{-\frac{t}{\tau}}\right) & t \geq 0
\end{array}\right.
$$

the potential looks like

$$
\varphi_{\uparrow}=\frac{\zeta c}{4 \pi} \frac{q_{0}}{2 r r_{0}}
$$

$$
\left\{\begin{array}{cl}
0 & t \leq \frac{r-r_{0}}{c}, \\
c t-c \tau+r_{0}-r+c \tau e^{\frac{r-r_{0}-c t}{c \tau}} & \frac{r-r_{0}}{c} \leq t \leq \frac{r+r_{0}}{c}, \\
2 r_{0}-c \tau\left(e^{\frac{r+r_{0}}{c \tau}}-e^{\frac{r-r_{0}}{c \tau}}\right) e^{-\frac{t}{\tau}} & \frac{r+r_{0}}{c} \leq t<\infty
\end{array}\right.
$$

The corresponding $\epsilon$-field for this potential is:

$$
\begin{aligned}
& \epsilon_{\uparrow}(r, t)=\frac{1}{c} \frac{\partial \varphi_{\uparrow}}{\partial t}=\frac{\zeta c}{4 \pi} \frac{q_{0}}{2 r r_{0}} \\
& \left\{\begin{array}{cl}
0 & t \leq \frac{r-r_{0}}{c}, \\
1-e^{\frac{r-r_{0}}{c \tau} e^{-\frac{t}{\tau}}} & \frac{r-r_{0}}{c} \leq t \leq \frac{r+r_{0}}{c}, \\
\left(e^{\frac{r+r_{0}}{c \tau}}-e^{\frac{r-r_{0}}{c \tau}}\right) e^{-\frac{t}{\tau}} & \frac{r+r_{0}}{c} \leq t<\infty,
\end{array}\right.
\end{aligned}
$$

The corresponding longitudinal electric field for this potential is:

$$
E_{r \uparrow}(r, t)=-\frac{\partial \varphi_{\uparrow}}{\partial r}=\epsilon_{\uparrow}(r, t)+\frac{\varphi_{\uparrow}}{r} .
$$

We can see that the $\epsilon$-field (34) has the form of a spherical layer - it is concentrated in a spherical layer with 
an external radius of $r=r_{0}+c t$ and width $c \tau$. This spherical layer propagates at the speed of light. As the spherical layer moves away from the charged shell the $\epsilon$-field decreases as $1 / r$. The electric field consists of two parts. One part has the same form as the $\epsilon$-field. This is a leading front of the electric field propagating away from the shell. The coulomb field which is formed behind this front is the second part of the electric field.

When the charge of the shell decreases from $q_{0}$ according to

$$
q(t)=\left\{\begin{array}{rl}
q_{0} & t \leq 0 \\
q_{0} e^{-\frac{t}{\tau}} & t \geq 0
\end{array}\right.
$$

its potential in the external region $r \geq r_{0}$ is given by

$$
\begin{gathered}
\varphi_{\downarrow}(r, t)=\frac{\zeta c}{4 \pi} \frac{q_{0}}{2 r r_{0}} \times \\
\left\{\begin{array}{cl}
2 r_{0} & t \leq \frac{r-r_{0}}{c}, \\
c \tau-c t+r_{0}+r-c \tau e^{\frac{r-r_{0}-c t}{c \tau}} & \frac{r-r_{0}}{c} \leq t \leq \frac{r+r_{0}}{c}, \\
c \tau\left(e^{\frac{r+r_{0}}{c \tau}}-e^{\frac{r-r_{0}}{c \tau}}\right) e^{-\frac{t}{\tau}} & \frac{r+r_{0}}{c} \leq t<\infty .
\end{array}\right.
\end{gathered}
$$

Such a vanishing charge creates the $\epsilon$-field

$$
\epsilon_{\downarrow}(r, t)=-\epsilon_{\uparrow}(r, t)
$$

and the longitudinal electric field

$$
E_{r \downarrow}(r, t)=-\frac{\partial \varphi_{\downarrow}}{\partial r}=\epsilon_{\downarrow}(r, t)+\frac{\varphi_{\downarrow}}{r} .
$$

The $\epsilon$-field created by a vanishing charge is the same spherical layer as the field of the charge being generated, but has an opposite sign. The electric field consists of two parts. One part is the same spherical layer as the $\epsilon$-field. This layer propagates away from a vanishing charge and "erases" its coulomb field. Behind the spherical layer the coulomb field has been erased, but it is still extant in front of the spherical layer. This is the second part of the electric field.

\subsection{Influence of the $\epsilon$-field on charges}

What is the effect of $\epsilon$-field on other charges? In order to answer this question let us turn the conservation laws of energy and momentum (57),(58). For (28)-(31) they are:

$$
\begin{gathered}
\frac{\partial}{\partial t}\left(\frac{E^{2}+c^{2} B^{2}+\epsilon^{2}}{2 \zeta c}\right)+(\boldsymbol{E} \boldsymbol{j}-\epsilon \rho c)=-\operatorname{div} \frac{\boldsymbol{E} \times \mathrm{c} \boldsymbol{B}+\epsilon \boldsymbol{E}}{\zeta} . \\
\frac{\partial}{\partial t} \frac{\boldsymbol{E} \times c \boldsymbol{B}-\epsilon \boldsymbol{E}}{\zeta c^{2}}+\left(\rho \boldsymbol{E}+\frac{1}{c} \boldsymbol{j} \times c \boldsymbol{B}-\frac{1}{c} \epsilon \boldsymbol{j}\right)=\nabla \cdot \mathrm{T} .
\end{gathered}
$$

The terms $\rho \boldsymbol{E}+\boldsymbol{j} \times \boldsymbol{B}-\epsilon \boldsymbol{j} / c$ in (40) describe forces which are exerted on the charges, and the terms $\boldsymbol{E} \boldsymbol{j}-$ $\epsilon \rho c$ in (39) describe the power of these forces. For a point charge $q$ these terms describe the rate of change of energy $\mathcal{E}=m c^{2} / \sqrt{1-v^{2} / c^{2}}$ and change of momentum $\boldsymbol{p}=m \boldsymbol{v} / \sqrt{1-v^{2} / c^{2}}$ of this charge:

$$
\frac{d \mathcal{E}}{d t}=q \boldsymbol{v} \cdot \boldsymbol{E}-q c \epsilon=q \boldsymbol{v} \cdot \boldsymbol{E}-\epsilon \mathcal{E} \frac{q}{m c} \sqrt{1-\frac{v^{2}}{c^{2}}},
$$

$\frac{d \boldsymbol{p}}{d t}=q \boldsymbol{E}+\frac{q}{c} \boldsymbol{v} \times c \boldsymbol{B}-\frac{q}{c} \epsilon \boldsymbol{v}=q \boldsymbol{E}+\frac{q}{c} \boldsymbol{v} \times c \boldsymbol{B}-\epsilon \boldsymbol{p} \frac{q}{m c} \sqrt{1-\frac{v^{2}}{c^{2}}}$.

As we can see $\epsilon$-field brings a contribution to the interaction. The contribution is significantly different from the contributions of the electric field $\boldsymbol{E}$ and magnetic field $\boldsymbol{B}$. Fields $\boldsymbol{E}$ and $\boldsymbol{B}$ rotate the 4-vector of energymomentum of the charged particle in Minkowski space without changing its magnitude. Conversely, the $\epsilon$-field, as we can see from (41)-(42), changes all coordinates of energy-momentum vector proportionally, i.e. changes magnitude of this vector. In other words, the $\epsilon$-field changes the mass of the affected charge. Let us derive an equation describing this change. In order to do this let us take product of (41) with $2 \mathcal{E}$, and the dot product of (42) with $2 \boldsymbol{p} c^{2}$, and subtract them:

$$
\begin{gathered}
\frac{d\left(\mathcal{E}^{2}-p^{2} c^{2}\right)}{d t}=\frac{d m^{2} c^{4}}{d t}= \\
=2 q\left(\mathcal{E} \boldsymbol{v} \cdot \boldsymbol{E}-c^{2} \boldsymbol{p} \cdot \boldsymbol{E}-c \boldsymbol{p} \cdot \boldsymbol{v} \times \boldsymbol{B}-c \mathcal{E} \epsilon+c \boldsymbol{p} \cdot \boldsymbol{v} \epsilon\right)= \\
-2 q c^{3} \epsilon m \sqrt{1-\frac{v^{2}}{c^{2}}} .
\end{gathered}
$$

This yields:

$$
\frac{d m c^{2}}{d t}=-q c \epsilon \sqrt{1-\frac{v^{2}}{c^{2}}}, \quad \text { or } \quad \frac{d m c^{2}}{d \tau}=-q c \epsilon .
$$

This is the rate of mass change of the charged particle by the interaction term $\epsilon j^{\mu}$.

For a system of charges distributed with density $\rho$, which is moving with velocity $v,(43)$ must be changed as follows:

$$
\frac{d m c^{2}}{d t}=-\int_{V} c \rho \epsilon \sqrt{1-\frac{v^{2}}{c^{2}}} d V
$$

Thus we find that a varying charge creates the $\epsilon$ field, which affects other charges and changes their rest masses. When a charge which is being created acts on a charge of the same sign, by means of the $\epsilon$-field, the rest mass of the second charge decreases. The rate of this decreasing is proportional to the rate of charge increasing and value of the charge exposed to the $\epsilon$-field.

At a glance such action of the $\epsilon$-field seems unusual. However, upon close consideration it turns out to be natural and resonable. Let us illustrate this by the example of a constant charge $q$, which interacts with a varying rest charge $q_{1}$. Charge energy change is expressed by (41):

$$
\frac{d \mathcal{E}}{d t}=q \boldsymbol{v} \cdot \boldsymbol{E}-q c \epsilon=-q \frac{\partial \varphi_{1}}{\partial \boldsymbol{r}} \frac{d \boldsymbol{r}}{d t}-q \frac{\partial \varphi_{1}}{\partial t}=-q \frac{d \varphi_{1}(\boldsymbol{r})}{d t},
$$

or

$$
\frac{m(t) c^{2}}{\sqrt{1-v^{2} / c^{2}}}+q \varphi_{1}(t, \boldsymbol{r})=\text { const. }
$$

One can see that any change in the potential energy of charge $q$ generated by interaction with $q_{1}$ is compensated by its energy change $m c^{2} / \sqrt{1-v^{2} / c^{2}}$. It becomes trivial if charge $q_{1}$ does not change and creates only the electric field. For example, charge $q$ at infinity moving towards $q_{1}$, with velocity $v$, approaches $q_{1}$ until its primary kinetic energy $m c^{2} / \sqrt{1-v^{2} / c^{2}}-m c^{2}$ becomes 
equal to potential energy $q \varphi_{1}$. However, for varying charge $q_{1}$, the potential energy of charge $q$ can change for reasons other than its motion relative to $q_{1}$. For example, increasing charge $q_{1}$ creates the fields $\epsilon$ and $\boldsymbol{E}$. While propagating they achieve charge $q$ which appears in the scope of influence of potential $\varphi_{1}$. Therefore its potential energy changes from zero to $q \varphi_{1}$. The rest and constant charge $q$ has only one possibility to compensate this change, in agreement with the conservation law of energy, - to change its rest energy and mass. This takes place under the influence of the $\epsilon$-field and is described by the terms $\epsilon \rho c$ and $\epsilon \boldsymbol{j} / c$ in (41) and (42).

\subsection{Radiation of energy by non- conserved charge}

We find that varying charge is at rest generate $\epsilon$ and $\boldsymbol{E}$ fields. These fields propagate away from the charge and carry some energy. According to (39) the flux density of this energy is equal to $\epsilon \boldsymbol{E} / \zeta$. As an example we will find the energy emitted by a spherical shell with a charge changing according to (33) or (36). We have already found the fields of such a charged spherical shell of radius $r_{0}$. Let us surround this shell by a spherical surface of radius $R\left(R \geq r_{0}\right)$ and calculate the energy flux through this surface for the time interval from 0 to $\infty$ :

$$
\int_{0}^{\infty} \frac{\epsilon E}{\zeta} 4 \pi R^{2} d t= \pm \frac{c \zeta}{4 \pi} \frac{q_{0}^{2}}{2 R}+\frac{c \zeta}{4 \pi} \frac{q_{0}^{2}}{2 r_{0}}\left[1-\frac{c \tau}{2 r_{0}}\left(1-e^{-\frac{2 r_{0}}{c \tau}}\right)\right]
$$

The sign "+" refers to the case of charge increasing (33), sign "-" refers to the case of charge decreasing (36).

The first term in (46) is equal in magnitude to the coulomb field energy in the range $R \leq r<\infty$ outside the surface $R$. The coulomb energy of the increasing charge propagates away from it and flows through the surface $R$ into the volume $r \geq R$ (the first component with the sign "+"). The coulomb energy from $r \geq R$ flows into the volume $r \leq R$ through the surface $R$ and goes back to the decreasing charge (the first component with the sign "-").

If $R \rightarrow \infty$ the first term tends to zero, i.e., the coulomb energy does not pass through an infinitely distant surface. It is "tied" to the charge and it is not emitted irretrievably.

The second term in (46) does not depend on surface radius $R$. It describes energy which is carried off irretrievably by the leading front impulses of the electric and $\epsilon$-fields. When these impulses pass through the surface of radius $R$ surrounding the charge, they carry energy through it described by the second component in (46). If the radius of the charged shell is small enough, such that $r_{0} \ll c \tau$, the second component tends to the value

$$
W_{\text {rad }}=\frac{\zeta c}{4 \pi} \frac{q_{0}^{2}}{2 c \tau}
$$

Such energy is emitted by the point charge when it increases or decreases according to the (33) or (36).

The natural question is: what is the source of the coulomb energy, which propagates away from the charge and disappears forever? In order to answer this question, let us investigate the energy balance while the charge is varying. For this purpose we use the integral form of
$(39)$ :

$$
\frac{d}{d t} \int_{V} \frac{E^{2}+\epsilon^{2}}{2 \zeta c} d V-\int_{V} \epsilon c \rho(\boldsymbol{r}) d V=-\int_{S} \frac{\epsilon \boldsymbol{E}}{\zeta} d \boldsymbol{S} .
$$

This equation expresses energy balance of stationary charge distribution during the change of $\rho$ in volume $V$, enclosed by the surface of radius $R$. Using (44), we find

$$
-\frac{d m c^{2}}{d t}=\frac{d}{d t} \int_{V} \frac{E^{2}+\epsilon^{2}}{2 \zeta c} d V+\int_{S} \frac{\epsilon \boldsymbol{E}}{\zeta} d \boldsymbol{S} .
$$

It is easy to see that the energy source of electric and $\epsilon$-fields is the rest energy of the particles with varying charges.

Let us apply (49) to the above example of the charged shell follow what happens in this case. Let us calculate the energy change due to (48) inside the spherical surface of radius $R$, enclosing the $r_{0}$ shell, during all the time of shell charge varying, from 0 to $\infty$.

Charge increases according to (33) create an $\epsilon$-field (34). This $\epsilon$-field affects shell charges and changes their rest energies according to (44)

$$
-\Delta m c^{2}=\int_{0}^{\infty}\left(\int_{V} c \rho \epsilon_{\uparrow} d V\right) d t=c \int_{0}^{\infty} q(t) \epsilon_{\uparrow}\left(r_{0}, t\right) d t=
$$

$$
=\frac{\zeta c}{4 \pi} \frac{q_{0}^{2}}{2 r_{0}}+\frac{\zeta c}{4 \pi} \frac{q_{0}^{2}}{2 r_{0}}\left[1-\frac{c \tau}{2 r_{0}}\left(1-e^{-\frac{2 r_{o}}{c \tau}}\right)\right] .
$$

While the shell charge increases to $q_{0}$ its primary rest energy $m_{0} c^{2}$ decreases by the amount given in (49a). Let us calculate varying energy of the fields. There are no fields inside the $R$ surface at $t=0$. There is the coulomb field between this surface and the $r_{0}$ surface when $t \rightarrow \infty$, which has an energy equal to $\frac{\zeta c}{4 \pi}\left(\frac{q_{0}^{2}}{2 r_{0}}-\frac{q_{0}^{2}}{2 R}\right)$. Moreover, the energy given in (46) has been emitted through the surface $R$. Thus, equation (48) integrated over time from 0 to $\infty$ takes on the form

$$
\begin{gathered}
\underbrace{\frac{\zeta c}{4 \pi} \frac{q_{0}^{2}}{2 r_{0}}+\frac{\zeta c}{4 \pi} \frac{q_{0}^{2}}{2 r_{0}}\left[1-\frac{c \tau}{2 r_{0}}\left(1-e^{-\frac{2 r_{o}}{c \tau}}\right)\right]}_{-\Delta m c^{2}}= \\
=\underbrace{\frac{\zeta c}{4 \pi}\left(\frac{q_{0}^{2}}{2 r_{0}}-\frac{q_{0}^{2}}{2 R}\right)}_{W_{\text {Coul }}}+ \\
\underbrace{\frac{\zeta c}{4 \pi} \frac{q_{0}^{2}}{2 r_{0}}\left[1-\frac{c \tau}{2 r_{0}}\left(1-e^{-\frac{2 r_{o}}{c \tau}}\right)\right]+\frac{\zeta c}{4 \pi} \frac{q_{0}^{2}}{2 R}}_{W_{\text {rad }}} .
\end{gathered}
$$

One can see that at charge birth its rest energy decreases, and due to this it acquires the energy of the coulomb field. I.e., the energy of the coulomb field, propagating away from a charge during its birth, is taken from its rest energy. Thus, the birth of a charge is the effective method of direct transformation of rest energy into electric energy. Moreover, at charge birth a finite part of rest energy is emitted irretrievably. The decrease of rest energy is exactly equal to the sum of the coulomb field energy and emitted energy. 
In the case when $r_{0} \ll c \tau, R \rightarrow \infty$, (50) becomes:

$$
\underbrace{\frac{\zeta c}{4 \pi} \frac{q_{0}^{2}}{2 r_{0}}+\frac{\zeta c}{4 \pi} \frac{q_{0}^{2}}{2 c \tau}}_{-\Delta m c^{2}}=\underbrace{\frac{\zeta c}{4 \pi} \frac{q_{0}^{2}}{2 r_{0}}}_{W_{\text {Coul }}}+\underbrace{\frac{\zeta c}{4 \pi} \frac{q_{0}^{2}}{2 c \tau}}_{W_{\text {rad }}} .
$$

When a charge disappears according to (36), its coulomb field disappears as well. The energy of this field between the surfaces of $r_{0}$ and $R$ decreases by the amount $\frac{\zeta c}{4 \pi}\left(\frac{q_{0}^{2}}{2 r_{0}}-\frac{q_{0}^{2}}{2 R}\right)$. As a result of the $\epsilon$-field (37) action on the shell charges, their rest energy increases by the amount:

$\Delta m c^{2}=-c \int_{0}^{\infty} q(t) \epsilon_{\downarrow}\left(r_{0}, t\right) d t=\frac{\zeta c}{4 \pi} \frac{q_{0}^{2}}{2 r_{0}} \frac{c \tau}{2 r_{0}}\left(1-e^{-\frac{2 r_{\mathrm{o}}}{c \tau}}\right)$.

Moreover, the energy given in (46) radiates through the surface $R$. The energy conservation law (48), integrated over time from 0 to $\infty$ is

$$
\begin{aligned}
& \underbrace{\frac{\zeta c}{4 \pi}\left(\frac{q_{0}^{2}}{2 r_{0}}-\frac{q_{0}^{2}}{2 R}\right)}_{-W_{\text {Coul }}}= \\
& =\underbrace{\frac{\zeta c}{4 \pi} \frac{q_{0}^{2}}{2 r_{0}} \frac{c \tau}{2 r_{0}}\left(1-e^{-\frac{2 r_{o}}{c \tau}}\right)}_{\Delta m c^{2}}+ \\
& \underbrace{\frac{\zeta c}{4 \pi} \frac{q_{0}^{2}}{2 r_{0}}\left[1-\frac{c \tau}{2 r_{0}}\left(1-e^{-\frac{2 r_{o}}{c \tau}}\right)\right]-\frac{\zeta c}{4 \pi} \frac{q_{0}^{2}}{2 R}}_{W_{\text {rad }}} .
\end{aligned}
$$

We can see that at charge disappearance its coulomb field energy transforms partly into rest energy and partly is emitted irretrievably. The decrease of the coulomb energy exactly equal the sum of rest energy increase and the emitted energy.

In the case when $r_{0} \ll c \tau$ and $R \rightarrow \infty$, (52) is simplified:

$$
\underbrace{\frac{\zeta c}{4 \pi} \frac{q_{0}^{2}}{2 r_{0}}}_{-W_{\text {Coul }}}=\underbrace{\frac{\zeta c}{4 \pi} \frac{q_{0}^{2}}{2 r_{0}}-\frac{\zeta c}{4 \pi} \frac{q_{0}^{2}}{2 c \tau}}_{\Delta m c^{2}}+\underbrace{\frac{\zeta c}{4 \pi} \frac{q_{0}^{2}}{2 c \tau}}_{W_{\text {rad }}} .
$$

The above transitions of energy from one form into another look like the transitions of energy in an electric circuit: the electric energy of a capacity transforms to the magnetic energy of inductance and vice versa. Part of the energy is emitted in each such transition. In our case (at the charge varying) rest energy passes to the coulomb field energy and vice versa. Part of the energy is emitted.

\subsection{Wigner's paradox}

It was noted by Wigner that invariance considerations, which substantiate the electric charge conservation law, are less convincing than those which substantiate the conservation laws of energy, momentum and angular momentum [23]. In this connection he tried to link conservation of charge with conservation of energy. For this purpose Wigner considered the following thought experiment. Let us consider the charged Faraday cage with potential $\varphi_{1}$ and create a charge $q$ inside it. Let the sign of the electric charge be the same as the sign of the cage charge. Some amount of energy $W$ will be used for this. Then we carry the charge into a point with potential $\varphi_{2}$, which is distant from the cage. The work which is done is $A=q\left(\varphi_{1}-\varphi_{2}\right)$. Now let us annihilate the charge. Thus we will return the same energy $W$, as in Maxwell electrodynamics none of the process depends on the actual potential value. Then we carry the particle, which is already uncharged, into a primary point inside the cage, without spending energy for this movement. During this closed cycle procedure we get work $A$, that contradicts the first law of thermodynamics. From this consideration Wigner concludes that the initial assumption about the possibility of charge creation is wrong. But this consideration contains an obvious logical error. Wigner's analysis of a process with a non-conserved charge is based on Maxwell electrodynamics, which describes processes with conserved charges only. It is not necessary to discuss thought experiments with non-conserved charges in the framework of Maxwell equations in order to get a contradiction with fundamental physical statements. The equation $\nabla \cdot \boldsymbol{E}=\zeta c \rho$ shows that at a quite distant point from the charge the electric field appears or disappears simultaneously with the appearance or disappearance of the charge. So, it propagates with infinite velocity.

But there is no contradiction if processes with the nonconserved charges are analyzed with the help of e (28)(31).

We can prove this by considering Wigner's circular process in the framework of (28)-(31). While the charge is being created its rest energy decreases by the amount $\Delta m c^{2}=W_{\text {Coul }}+W_{\text {rad,1 } 1}$. This energy is used in order create the energy of the coulomb field $W_{\text {Coul }}$ and field of radiation $W_{\text {rad,1 } 1}$. Besides this, when the $\epsilon$-field, which appeared at the moment of charge birth reaches the surface of Faraday cage, its rest energy decreases by the amount $\Delta M c^{2}=q \varphi_{1}$. This follows from the fact that the cage section, having a charge $d Q$ and being at a distance of $r_{q, d Q}$ from the charge $q$, decreases its rest energy by the amount $d Q \cdot(\zeta c) /(4 \pi) \cdot q /\left(r_{q, d Q}\right)$ according to $(45)$. The entire cage decreases its rest energy by the amount

$$
q \frac{\zeta c}{4 \pi} \int_{0}^{Q} \frac{d Q}{r_{q, d Q}}=q \varphi_{1} .
$$

Here $\varphi_{1}$ is the potential created by the cage at location of charge $q$. While transferring the charge to a point with potential $\varphi_{2}$ we get the work $A=q\left(\varphi_{1}-\varphi_{2}\right)$. At annihilation of the charge a part of the energy of its Coulomb field $W_{\text {Coul }}$ is transformed into radiation energy of $W_{\text {rad,2 }}$, and the other part $W_{\text {Coul }}-W_{\text {rad,2 }}$ is transformed into rest energy and thus $m c^{2}-W_{C o u l}-$ $W_{\text {rad }, 1}+\left(W_{\text {Coul }}-W_{\text {rad }, 2}\right)=m c^{2}-W_{\text {rad }, 1}-W_{\text {rad }, 2}$. When the $\epsilon$-field, which appears at the charge annihilation, reaches the surface of cage, it initiates the increase of its rest energy by $q \varphi_{2}$ and makes it equal to $M c^{2}-q \varphi_{1}+q \varphi_{2}$. Now we can return the uncharged particle into its primary position without spending energy. As a result of completion of the cycle, rest energy of a particle decreases by $W_{\text {rad }, 1}+W_{\text {rad,2 }}$, and the rest energy of the cage decreases by $q\left(\varphi_{1}-\varphi_{2}\right)$. The first decrease $\left(W_{\text {rad }, 1}+W_{\text {rad }, 2}\right)$ is spent for radiation of two $\epsilon$-impulses at the birth and annihilation of the charge. 
The second $\left(q \varphi_{1}-q \varphi_{2}\right)$ - for the work of moving the charge between potentials $\varphi_{1}$ and $\varphi_{2}$. As we can see, the supposition about birth and annihilation of a charge, discussed by (28)-(31), does not contradict the energy conservation law.

\subsection{Electromagnetic mass and renor- malization}

As was shown in the previous section, a non-conserved electric charge does not result in the non-conserving of energy. As the charge varies its mass changes in a way that total energy (rest energy, coulomb field energy and energy of radiation) remains constant. Hence, at charge birth, rest energy is the source of field energy. It is not obvious that energy conservation must be provided exactly in this way. It is possible to expect that energy of the fields is created at the expense of energy of the external agent creating the charge. This is the exact Wigner's [23] supposition . However, equations (28)(31) are arranged in such a way that they don't require any external agent. According to (50) the non-field mass $m_{e}$ of the born charge is less than the primary mass $m_{0}$ of the uncharged particle:

$$
m_{e}=m_{0}-\frac{W_{\text {Coul }}}{c^{2}}-\frac{W_{\text {rad }}}{c^{2}} .
$$

The non-field mass $m_{e}$ is the remainder of the primary mass $m_{0}$. A part of the primary mass transforms into electromagnetic mass, which is equivalent to the coulomb field energy, and into radiation energy. Total charge mass (non-field and electromagnetic) is equal to:

$$
m_{e}+\frac{W_{\text {Coul }}}{c^{2}}=m_{0}-\frac{W_{\text {rad }}}{c^{2}} .
$$

When the radius of the charge tends to zero, $W_{\text {Coul }}$ and $m_{e}$ tend to infinity but their sum (total mass) remains finite and less than the primary mass of the uncharged particle, because a part of this mass transforms into energy of radiation.

Relationship (55) is similar to that used for renormalization of mass. The renormalization hypothesis assumes that observable mass of the charged particle $m$ is the sum of the "naked" unobservable mass $m_{e}$ and electromagnetic mass, and this sum is a finite quantity:

$$
m=m_{e}+\frac{W_{\text {Coul }}}{c^{2}} .
$$

As we can see from the model described by (28)-(31), such a relationship arises quite natural and consistently, but not as a supposition. The renormalization mechanism is not brought from the outside, it is inherent to equations (28)-(31), which describe the processes of charge varying. Relationship (55) can be treated as substantiation of the hypothesis of renormalization in classical electrodynamics. It links the finite observable mass of the charge with the primary mass of the uncharged particle. At the same time, relationship (44) may be treated as a dynamic realization of the renormalization hypothesis. It describes the process of mass transference from the non-field form into the field form and vice versa, at charge birth and annihilation.

From the above consideration it follows quite certainly, that mass $m$ from equations of motion (41),(42), decreases at charge birth and increases at its annihilation. Therefore, it is the non-field mass. Thus, these equations do not take into account the contribution of electromagnetic mass into the charge inertia. However, there is no doubt that the electromagnetic energy of the charge contributes to its inertia. So the question arises: in what way must this contribution be taken into account in the equations of motion? We also remember that (41),(42) do not take into account the influence of their own field of radiation (radiation reaction) on the charge motion. Probably, both effects must be taken into account by the same mechanism: by introducing, into the equations of motion, the self-reaction force of the $\boldsymbol{E}$ and $\boldsymbol{B}$ fields onto the charge creating these fields. Particle mass $m$ from the equations of motion (41),(42) decreases when its charge appears. Also, self-reaction forces must appear in these equations. According to the Lorentz [24] supposition, self-action of a coulomb field must change the inertial properties of the charge and in a such way compensate for the decreasing of the non-field mass. Therefore, the contribution of electromagnetic mass must be taken into account by means of the self-action of the coulomb field. The self-action of the transversal field must take into account the radiation reaction. This is an old problem of electrodynamics, which is not solved yet. Repeated attempts (for example, $[25,26,27])$ to take self-action into account have failed. Our consideration allowed us to take into account the self-action of the $\epsilon$-field and to find out that this self-action results in mutual transformations of the field and non-field masses at charge varying. However, the problem of the self-action of the $\boldsymbol{E}$ and $\boldsymbol{B}$ fields remains unsolved. A different method of accounting for the self-action of the coulomb field is proposed by renormalization. It is introducing, into the equations of motion, the complete observable mass instead of the nonfield mass and self-action of coulomb field. It should be emphasized that renormalization is in no way connected with the infinity of electromagnetic mass for a point charge. Even if a method to make this mass finite is found, the problem of including it into the equations of motion remains.

\section{Discussion}

The natural question is if it is really necessary to investigate processes with non-conserved electric charge, when it is indeed conserved in all known processes? There are as least two reasons for doing this.

The first reason is that such research helps us to understand better even those questions which are not directly connected to charge varying. Thus, for example, we find that there is a mechanism of mutual transformations of rest energy and electric energy, which allows a new look at the problem of renormalization and selfaction. For this reason it is important to extend the research of non-conserved charge processes into the scope of quantum electrodynamics. It is interesting because in QED the Lorentz condition is executed only on average. As a consequence the $\epsilon$-field is also eliminated only on average, $\left\langle\psi\left|\partial_{\mu} A^{\mu}\right| \psi\right\rangle=\langle\psi|\epsilon| \psi\rangle=0$. But it does not eliminate the possibility of occurrence of the $\epsilon-\boldsymbol{E}$-field 
fluctuation effects.

The second reason is purely speculative. One can suppose the violation of the electric charge conservation law in the area of energies higher than attained now, and also in the early stages of universe development. If processes with charge varying are found at high energies they will become the effective method of rest energy transformation into electric energy. Also, it is not necessary to assume that elementary particles initially appeared in their present form at the early stages of the development of the universe. It is possible that originally matter was maximally homogeneous and particles had only one property - their masses. Subsequently differentiation took place, and particles gain charges, spending part of their rest energy on the energy of field creation.

\section{Conclusions}

In this article we make the first step in research of the hypercomplex Dirac equation. Already this first research demonstrates interesting and unusual properties of this equation. In particular, this equation contains the electrodynamics of non-conserved charges and describes the interaction of such charges. These interaction are highly unusual: they cause changes of rest mass and can be responsible for the mechanism of renormalization of electric charge masses. Equations (8)-(15) can be used for the description of the massive vector bosons, produced by non-conserved charges (for example, $\mathrm{W}$ and $\mathrm{Z}$ bosons).

As it can be seen from (8)-(15) that propagators of the bosons must look like:

$$
D \sim \frac{1}{k^{2}-\varkappa^{2}}
$$

which provide re-normalization of the theory.

\section{A Appendix}

\section{A.1 Conservation laws}

Combining the (8)-(15), it is possible to get the conservation laws of energy and momentum as in the Maxwell electrodynamics. To get the conservation law of energy, let us form a combination from (8)-(15)

$$
\begin{aligned}
\epsilon \times(8)+\boldsymbol{E} \times(9) & -\beta \times(10)+ \\
c \boldsymbol{B} \times(11)+V^{0} \times(12) & +\boldsymbol{V} \times(13)+ \\
\boldsymbol{U} \times(14) & +U^{0} \times(15) .
\end{aligned}
$$

After dividing by $\zeta$ we find

$$
\begin{array}{r}
\frac{1}{c} \frac{\partial}{\partial t} \frac{E^{2}+c^{2} B^{2}+\epsilon^{2}+\beta^{2}}{2 \zeta}+ \\
\left(\boldsymbol{E} \cdot \boldsymbol{j}_{e}-\epsilon c \rho_{e}-c \boldsymbol{B} \cdot \boldsymbol{j}_{m}-\beta c \rho_{m}\right)+ \\
+\frac{1}{c} \frac{\partial}{\partial t} \frac{V^{2}+U^{2}+\left(V^{0}\right)^{2}+\left(U^{0}\right)^{2}}{2 \zeta}+ \\
\left(\boldsymbol{V} \cdot \boldsymbol{k}-\boldsymbol{U} \cdot \boldsymbol{l}-V^{0} s+U^{0} p\right)= \\
=-\operatorname{div} \frac{\boldsymbol{E} \times c \boldsymbol{B}+\epsilon \boldsymbol{E}-\beta c \boldsymbol{B}}{\zeta}- \\
\operatorname{div} \frac{V^{0} \boldsymbol{V}+U^{0} \boldsymbol{U}+\boldsymbol{V} \times \boldsymbol{U}}{\zeta} .
\end{array}
$$

The sense of this relationship is obvious. Terms under $\partial / \partial t$ are the densities of energy of the fields, terms with charges and currents are the rates of change of energy of particles interacting with the fields. In other words, it is the power of forces with which the fields affect charges and currents. Terms under the sign of divergence are the densities of energy flux being carried by the fields.

To get the conservation law of momentum, let us form the following combination from (8)-(15)

$$
\begin{aligned}
\boldsymbol{E} \times(8)+c \boldsymbol{B} \times(9)+c \boldsymbol{B} \times(10) & -\boldsymbol{E} \times(11)+ \\
\epsilon \times(9)-\beta \times(11)+\boldsymbol{V} \times(14) & -\boldsymbol{U} \times(13)- \\
\boldsymbol{V} \times(12)-V^{0} \times(13)-U^{0} \times(14) & -\boldsymbol{U} \times(15) .
\end{aligned}
$$

Dividing by $c \zeta$ yields:

$$
\begin{array}{r}
\frac{1}{c} \frac{\partial}{\partial t} \frac{\boldsymbol{E} \times c \boldsymbol{B}-\epsilon \boldsymbol{E}+\beta c \boldsymbol{B}}{c \zeta}+ \\
+\frac{1}{c}\left(c \rho_{e} \boldsymbol{E}+\boldsymbol{j}_{e} \times c \boldsymbol{B}-c \rho_{m} c \boldsymbol{B}+\right. \\
+\frac{1}{c} \frac{\partial}{\partial t} \frac{\boldsymbol{U} \times \boldsymbol{V}+V^{0} \boldsymbol{V}+U^{0} \boldsymbol{U}}{c \zeta}+ \\
+\frac{1}{c}\left(-s \boldsymbol{V}+p \boldsymbol{U}+V^{0} \boldsymbol{k}-U^{0} \boldsymbol{l}+\right. \\
\boldsymbol{U} \times \boldsymbol{k}+\boldsymbol{V} \times \boldsymbol{l})=\nabla \cdot \mathrm{T}
\end{array}
$$

Here $\nabla \cdot \mathrm{T}$ is a divergence of the stress tensor

$$
\begin{array}{r}
\nabla \cdot \boldsymbol{T}= \\
\frac{1}{c \zeta}[\boldsymbol{E} \nabla \cdot \boldsymbol{E}-\boldsymbol{E} \times \nabla \times \boldsymbol{E}+c \boldsymbol{B} \nabla \cdot c \boldsymbol{B}- \\
c \boldsymbol{B} \times \nabla \times c \boldsymbol{B}-\nabla \times(\epsilon c \boldsymbol{B})- \\
\nabla \times(\beta \boldsymbol{E})+\epsilon \nabla \epsilon+\beta \nabla \beta]+ \\
+\frac{1}{c \zeta}[-\boldsymbol{V} \nabla \cdot \boldsymbol{V}+\boldsymbol{V} \times \nabla \times \boldsymbol{V}-\boldsymbol{U} \nabla \cdot \boldsymbol{U}+ \\
\boldsymbol{U} \times \nabla \times \boldsymbol{U}+\nabla \times\left(V^{0} \boldsymbol{U}\right)-\nabla \times\left(U^{0} \boldsymbol{V}\right)- \\
\left.V^{0} \nabla V^{0}-U^{0} \nabla U^{0}\right] .
\end{array}
$$

The stress tensor is defined as

$$
\begin{array}{r}
T^{i j}= \\
\frac{1}{\zeta c}\left[E^{i} E^{j}+c^{2} B^{i} B^{j}-\frac{\eta^{i j}}{2}\left(E^{2}+c^{2} B^{2}-\epsilon^{2}-\beta^{2}\right)-\right. \\
\left.\varepsilon^{i j k}\left(\beta E_{k}+\epsilon c B_{k}\right)\right] \\
-\frac{1}{\zeta c}\left[V^{i} V^{j}+U^{i} U^{j}+\varepsilon^{i j k}\left(U^{0} V_{k}-V^{0} U_{k}\right)-\right. \\
\left.\frac{\eta^{i j}}{2}\left(V^{2}+U^{2}-\left(V^{0}\right)^{2}-\left(U^{0}\right)^{2}\right)\right] .
\end{array}
$$

In (58) terms under $\partial / \partial t$ are the densities of the momentum of the fields, terms with charges and currents are the densities of forces, with which the fields affect charges and currents. The stress tensor determines the density of the momentum flux carried by the fields. 
Our attention should be paid to the fact that the energy-momentum-stress tensor contains antisymmetric terms. However, it can always be made symmetric by building an equivalent metrical energy-momentumstress tensor.

\section{Acknowledgments}

The authors would like to thank Prof. Lukyanets S.P., Prof. Lev B.I., Prof. Tomchuk P.M. and Prof. Cooney for stimulating discussions.

\section{REFERENCES}

[1] C. G. Darwin, The wave equation of the electron, Royal Society of London Proceedings, Series A 118, 654 - 680, (1928).

[2] O. Laporte and G. E. Uhlenbeck, Application of spinor analysis to the Maxwell and Dirac equations, Phys. Rev. 37, 1380 - 1397, (1931).

[3] J. R. Oppenheimer, Note on Light Quanta and the Electromagnetic Field, Phys. Rev. 38, 725 - 746, (1931).

[4] W. Heisenberg and W. Pauli, Zur Quantendynamik der Wellenfelder, Zeitschrift fur Physik, 56, 1 - 2, (1929).

[5] R. Feynman, R. Leighton, M. Sands. The Feynman lectures on physics, Vol. 2, Addison-Wesley Pub. Comp., Massachusetts, 1963.

[6] A. M. Solunin, On a generalization of the equations of electrodynamics. Russian Physics Journal, Vol. 15, \# 7, 1017 - 1020, (1972).

[7] V. I. Ogievetsky, I. V. Polubarinov. Interacting Fields of Spin 1 and Symmetry Properties, Ann. Phys. 25, 358386 (1963).

[8] V. I. Ogievetsky, I. V. Polubarinov, Interacting Field of Spin 2 and the Einstein Equations, Ann. Phys. 35, 157-208 (1965).

[9] S. Weinberg, Photons and Gravitons in S Matrix Theory: Derivation of Charge Conservation and Equality of Gravitational and Inertial Mass, Phys. Rev. 135, B1049B1056 (1964).

[10] L. B. Okun, Y. B. Zeldovich, Paradoxes of Unstable Electron, Phys. Lett. B 78, 597-600 (1978).

[11] L. B. Okun, M. B. Voloshin, On the Electric Charge Conservation, JETP Lett. 28, 145-149 (1978).
[12] L. B. Okun, Tests of Electric Charge Conservation and the Pauli Principle, Sov. Phys. Usp. 32, 543-547 (1989).

[13] A. Y. Ignatiev, V. A. Kuzmin, M. E. Shaposhnikov, Is The Electric Charge Conserved?, Phys. Lett. B 84, 315-318 (1979).

[14] M. I. Dobroliubov, A. Y. Ignatiev, Millicharged Particles, Phys. Rev. Lett. 65, 679-682 (1990).

[15] A. Y. Ignatiev, G. C. Joshi, Possible Electric Charge Nonconservation and Dequantization in $\mathrm{SU}(2) \mathrm{U}(1)$ Models with Hard Symmetry Breaking, Phys. Lett. B 381, 216-220 (1996).

[16] S. Nussinov, Charge Nonconserving Decays, Phys. Rev. Lett. 59, 2401-2404 (1987).

[17] R. N. Mohapatra, S. Nussinov, Electric Charge Nonconservation and Minicharged Particles: Phenomenological Implications, Int. J. Mod. Phys. A 7, 3817-3834 (1992).

[18] Y. Aharonov, F. T. Avignone, III, R. L. Brodzinski, J. I. Collar, E. Garca, H. S. Miley, A. Morales, J. Morales, S. Nussinov, A. Ortiz de Solorzano, J. Puimedon, J. H. Reeves, C. Saenz, A. Salinas, M. L. Sarsa, and J. A. Villar, New Laboratory Bounds on the Stability of the Electron, Phys. Rev. D 52, 3785-3792 (1995).

[19] M. M. Tsypin, Longitudinal Photons and Conservation of Charge, Sov. J. Nucl. Phys. 50, 269-274 (1989)

[20] V. I. Ogievetsky, I. V. Polubarinov, The Notoph and its Possible Interactions, Sov. J. Nucl. Phys. 4, 156-161 (1967).

[21] N, Kemmer, On the Particles of Spin-1, Helv. Phys. Acta 33, 829-838 (1960).

[22] B. I. Lev, A probable approach to the geometrization of interaction, Modern Physics Letters A 3, N 10, 1025 - 1031, (1988).

[23] E. Wigner, Invariance in physical theory, Proc. Amer. Phil. Soc. 93, 521 - 526, (1949).

[24] H. Lorentz, The Theory of Electrons and Its Applications to the Phenomena of Light and Radiant Heat, Dover Publications Inc, New York, (1952).

[25] P. A. M. Dirac, Classical theory of radiating electrons, Proc. Roy. Soc. London A 167, 148 - 169, (1938).

[26] J. A. Wheeler and R. P. Feynman, Interaction with the absorber as the mechanism of radiation, Rev. Mod. Phys.17, 157 - 181, (1945).

[27] J. A. Wheeler and R. P. Feynman, Classical Electrodynamics in terms of direct interparticle action, Rev. Mod. Phys. 21, 425 - 433, (1949). 